\title{
INTERMITTENT BLADDER URINARY RETENTION IN A YOUNG WOMAN. AN UNUSUAL PRESENTATION OF PARTIAL URETHRAL DUPLICATION
}

\author{
GIANCARLO SARNELLI, LEONARDO D’URSO, GIOVANNI MUTO \\ Department of Radiology (GS) and Department of Urology (LDU, GM), S. Giovanni Bosco Hospital, \\ Turin, Italy
}

\begin{abstract}
A singular case of female accessory urethra associated to a mobile bladder stone with an unusual clinical presentation is reported. The role of perineal ultrasound is emphasized as a useful diagnostic tool to study female urethra in a static and dynamic setting, including cases of partial or complete urethral duplication.
\end{abstract}

Key words: urethra; abnormalities; urethral obstruction; bladder calculi

Int Braz J Urol. 2005; 31: 259-61

\section{INTRODUCTION}

Urethral duplication and accessory urethra are both extremely rare lower urinary tract malformations, especially in females. The exact etiology of these conditions is still unknown and among the reported classifications, the Ortolano-Nasarallah's one seems to be the more complete at this moment (1).

By a review of the literature, there is a general agreement on the higher incidence of urethral malformations in males and that in most cases the duplicated or accessory urethra lies on a sagittal ventral plane and joins the primary urethra in $70 \%$ of the cases (2-3). In females, the urethral duplication seldom lies on a coronal plane and it is very often associated with bladder duplication. It is not rare that associated upper urinary tract malformations occur (e.g. ureteral ectopia).

We report a singular case concerning a young woman with a mobile bladder stone indwelled in an accessory blind urethra placed on the coronal plane.

\section{CASE REPORT}

A 29 years-old woman came to our observation for recurrent bladder urinary retentions. Medical history showed since childhood mild dysuria and recurrent lower urinary tract infections. Because of these symptoms the patient was submitted to ultrasonography and a bladder stone (width $1 \mathrm{~cm}$ ) was diagnosed, in 1997. A subsequent urethrocystoscopy failed to demonstrate the calculus.

Because of recurrent bladder urinary retentions, in 2003 the patient underwent excretory urography that failed to show any urinary tract abnormalities.

In February 2004, a perineal ultrasound was carried out. It showed 2 linear hypoecoic separate structures coming from the bladder trigone up to the perineum. These 2 structures' pathways were parallel on a latero-lateral plane with the right not reaching the perineum (Figure-1).

An oval stone $1 \mathrm{~cm}$ wide indwelled in the bottom of the right structure while the second structure, situated on the left, had a normal course up to 


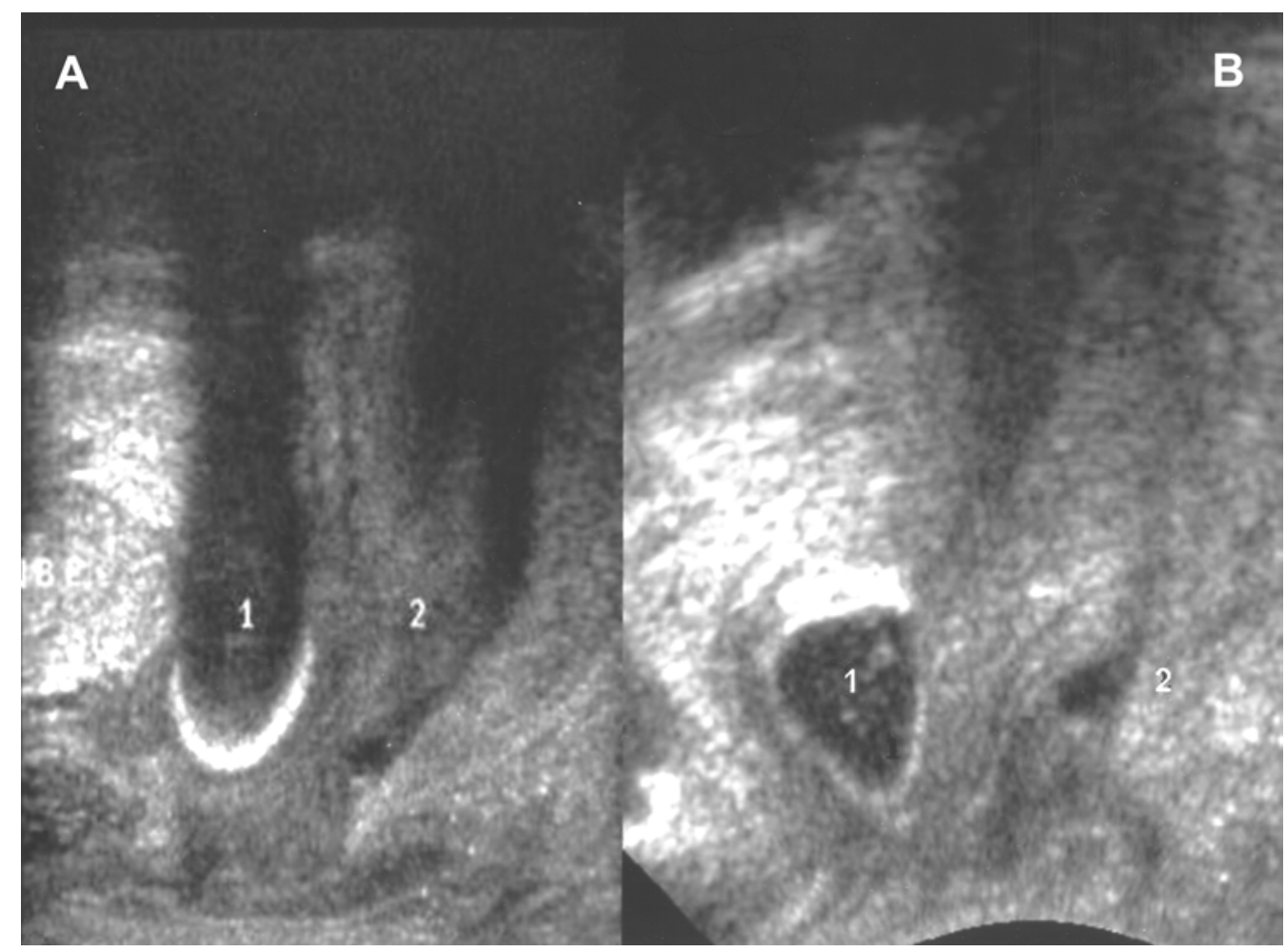

Figure 1 - Perineal ultrasound. A) Stone nested in the bottom of the blind accessory urethra [1] running parallel to primary urethra [2]. B) After micturition, stone is no more detectable because of its migration into the bladder. Accessory urethra's cul-de-sac [1] is still detectable.

external urethral meatus, with a small diverticulum in its middle. The bladder was normal.

A dynamic perineal ultrasound in supine position was particularly useful to show during the micturition the emptying of the accessory urethra and the migration of the stone into the bladder. In orthostatic position, the stone nested again in the accessory urethra.

A retrograde cystourethrography demonstrated an accessory blind urethra and, parallel to it on the left, the primary urethra with a severe prestenotic dilatation during micturition (Figure-2). After the micturition, the contrast medium outlined the bottom of accessory urethra and a small mid-urethral diverticulum of the primary urethra.

A urethrocystoscopy and mechanical cystolitotripsy was carried out and it was reported a substenotic external urethral meatus and normal bladder capacity with topic ureters.

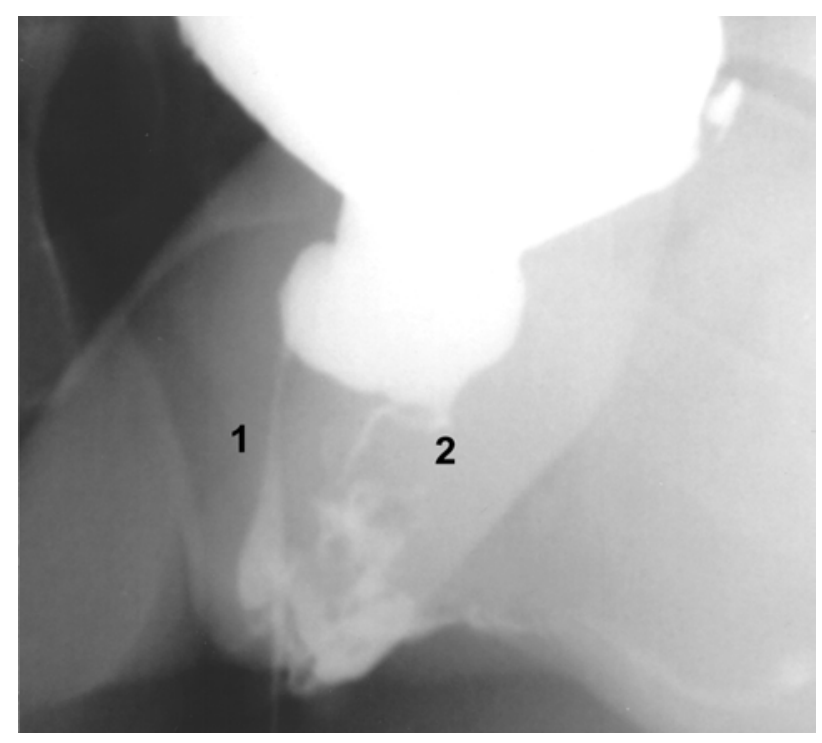

Figure 2 - Voiding cystography. On the left is evident accessory urethra distally ending in a cul-de-sac close to perineum [1]. A kinking of the primary urethra with a severe prestenotic dilatation is also evident [2]. 


\section{COMMENTS}

In a Medline literature review, similar cases were not found, in particular with reference to a partial or complete urethral duplication on a coronal plane.

The relevance of this case report is due to simultaneous multiple lower urinary tract malformations (accessory urethra, primary urethral diverticulum, and primary urethral substenosis) without any significant impact on the quality of life.

Of particular significance is the clinical behavior of the migrating bladder stone indwelled in the blind accessory urethra intermittently causing outlet obstruction. The small midurethral diverticulum of the primary urethra is probably secondary to mild-moderate urinary obstruction.

\section{REFERENCES}

1. Ortolano V, Nasrallah PF: Urethral duplication. J Urol. 1986; 136: 909-12.

2. Kennedy HA, Steidle CP, Mitchell ME, Rink RC: Collateral urethral duplication in the frontal plane: a spectrum of cases. J Urol. 1988; 139: 332-4.

3. Prasad N, Vivekanandhan KG, Ilangovan G, Prabakaran S: Duplication of the urethra. Pediatr Surg Int. 1999; 15: 419-21.

Received: January 1, 2005

Accepted after revision: February 10, 2005

\author{
Correspondence address: \\ Dr. Giancarlo Sarnelli \\ Dept. of Radiology, S. Giovanni Bosco Hospital \\ Piazza Donatore di Sangue, 3 \\ Turin, 10154, Italy \\ Fax: + 390112402456 \\ E-mail: giancarlosarnelli@libero.it
}

